\title{
Integration of Equation Solving Software with Data Acquisition Equipment for a Senior Engineering Measurements Course
}

\author{
David L. Walters \\ Mechanical Engineering Section \\ United States Coast Guard Academy
}

\begin{abstract}
The concomitant development of computer-based data acquisition systems, equation solving and spreadsheet software, and multimedia presentation software has provided engineering educators with a plethora of opportunities to positively and creatively impact course content, pedagogy, and student interest. This paper provides an account of a laboratory experience at the United States Coast Guard Academy,(USCGA) in utilizing National Instruments LabView data acquisition equipment, Excel and Mathcad analysis software, and Power Point presentational software in a Macintosh environment in the instruction of a senior level engineering measurement course.
\end{abstract}

The curriculum of the newly accredited Mechanical Engineering major at the USCGA not only emphasizes the design and open-ended problem solving elements necessary to an excellent engineering education, but seeks to augment the communications and leadership skills which the entire academy has embraced as desired outcomes. Experimental Methods in Fluid and Thermal Sciences is a senior level capstone experience in which the students receive classroom instruction in measurement systems and analysis techniques and apply these techniques in a three-hour weekly laboratory. Students typically work in laboratory groups of three and are required to design a measurement procedure and analysis plan. Students are encouraged to include a discussion of the strengths and weaknesses of their approach in their weekly written reports. Finally, students research a problem of interest, design a measurement system, conduct the experiment and report their findings in oral and written form as their project requirement.

The integration of different software packages in the acquisition, analysis and presentation of data has multiple positive effects which address the USCGA needs. The instructor can model the data acquisition, analysis and presentation process, and more effectively integrate graphics, equations and physical demonstrations to reach diverse learning styles. The efficiency of data acquisition and analysis facilitates proper statistical treatment of the data, immediate review of data and experimental procedures, inclusion of a broader and more interesting choice of experiments, and the interaction with students in the role of guide and mentor.

\section{INTRODUCTION}

The Experimental Methods in Fluid and Thermal Sciences (EMFTS) course is a senior level capstone experience for cadets at the United States Coast Guard Academy (USCGA). The course is designed to provide the students with a combination of lecture and laboratory experiences covering the topics of data acquisition, statistical analysis, Fourier analysis, calibration, operation of transducers, and documentation and communication of results. This paper documents efforts to augment the EMFTS course through the design of supplemental lecture and laboratory materials and exercises. 
During the Fall semester of 1996, EMFTS was cooperatively taught; lectures and laboratory design were created by LCDR V. Wilczynski of the USCGA, while the author conducted laboratory sessions, and augmented laboratory material through the design of presentation and analysis modules. Laboratories were scheduled during two sessions each week to accommodate the entire class. The thirteen weekly laboratory sessions included student oral presentations, laboratories, demonstrations, and culminated with an open ended student measurement project. Typically, the students worked in groups of two to four, and produced technical reports, memoranda or oral presentations as documentation, including a Power Point based presentation of their projects. The topic of this paper is to document the design of the laboratory and analysis modules developed during this course.

\section{DISCUSSION}

During the Fall semester 1996, EMFTS was taught to seniors for the first time at USCGA. Prior to this, it had been a junior level course. At the senior level, the course provides an opportunity for students to gain practical experience in the design, conduct and analysis stages of open ended measurement problems, just prior to a major effort in their Senior Design Projects in the Spring. The modules developed here were generated using Mathcad and were intended to augment the laboratory portion of the class, to be used as instructional tools to model proper experimental planning and data manipulation, and to stimulate student interest. These Mathcad routines or documents were used in two modes: most frequently, the document was displayed "live" on a large screen monitor, in conjunction with National Instruments LabView Data Acquisition software and equipment to demonstrate data acquisition and analysis, but portions of the documents were also used in Power Point slides for formal presentation as lecture notes.

Previous experience in the instruction of engineering courses using Mathcad (Ref 1) had been positive and enriching. However, there were three intrinsic difficulties or limitations of this approach in the EMFTS course. First, the students did not have Mathcad available to them, and so, the direct relevance, whether perceived and real, of the Mathcad documents to the their conduct of the experiments was vague. The students worked in Excel, so that any demonstration of procedure or approach presented in Mathcad required interpretation for implementation in Excel. Second, care had to be taken not to allow the elegance and simplicity of the data acquisition and analysis system to mask the details of the mathematics or data acquisition. Finally, the Mathcad documents were created during the course, so modifications were frequency, resulting in times when one section of students would be exposed to a different version of the document. Modifications were easily made during class or just prior to class, so that care had be taken to share "improvements" with each section of students.

The primary advantages to the approach were in the areas of modeling thoughtful analysis planning, and in providing additional insight into the data acquisition process. Analysis planning was modeled by presenting the relevant equations in a Mathcad document. Equations and necessary graphs were arranged in a logical order to achieve the desired output, and to accommodate the spreadsheet nature of Mathcad, which requires the sequential evaluation of the document. Outputs could then be easily examined prior to additional data acquisition. This process models the logic of an algorithm. Insight into the acquisition process was also enhanced through examination of the actual ASCII data files using Teach Text. Students were able to write data to a file using LabView, and immediately examine the data file using Teach Text.

The following modules are offered as examples of the power and elegance afforded the engineering educator through the marriage of LabView and Mathcad. These modules are presented in roughly the chronological order in which they were generated and presented to the students. 


\section{Outlier Detection}

The most difficult issue in developing and presenting an outlier detection module was in the generation of outlier data. The approach used here was to sample a strain gauge attached to an oscillating cantilevered beam. The data was put through a high-pass Butterworth filter within the LabView software which resulted in some larger voltage spikes due to the filter response. This approach was artificial, but provided the opportunity for the students to become familiar with writing data to a file using LabView and transferring the data file to Excel. Unfortunately, it became far too easy to generate and examine more data then was necessary to communicate the concepts. A more meaningful approach may be to use a contrived data file, such as an exam grade file as is demonstrated below.

First, data was read from a LabView generated data file, or, as in this case, from an ASCII file imported from another application.

$$
\mathrm{x}:=\operatorname{READPRN}(\text { data })
$$

The total number of data points was determined from the file, an index was established, and the mean calculated.

$$
\begin{aligned}
& \mathrm{N}:=\operatorname{last}(\mathrm{x}) \\
& \mathrm{x}_{\mu}:=\frac{\sum_{\mathrm{i}} \mathrm{x}_{\mathrm{i}}}{\mathrm{N}}
\end{aligned}
$$

The calculated mean was compared with the intrinsic function to provide feedback to the student and the process repeated with the standard deviation.

Next, the modified three-sigma test was applied. The z-variable and two-sided probability were calculated

$$
z_{o_{i}}:=\left|\frac{x_{i}-x_{\mu}}{S_{x}}\right| P\left(z_{0}\right):=\frac{2}{\sqrt{2 \cdot \pi}} \cdot \int_{0}^{z_{o_{i}}} e^{-\frac{\beta^{2}}{2}} d \beta
$$

at each point, the test applied and the data sorted.

$$
\begin{gathered}
\mathrm{a}_{\mathrm{i}}:=\mathrm{N} \cdot\left(1-\mathrm{P}\left(\mathrm{z}_{\mathrm{o}}\right)\right) \quad \mathrm{G}_{\mathrm{i}}:=\operatorname{if}\left(\mathrm{a}_{\mathrm{i}} \leq 1,0, \mathrm{x}_{\mathrm{i}}\right) \\
\mathrm{C}:=\operatorname{reverst}(\operatorname{sort}(\mathrm{G}))
\end{gathered}
$$

A comparison of the original data and the "processed" data was made using Mathcad's histogram feature. The accompanying graph shows the original data as histogram bars with the processed data superimposed as points. Note the ease with which the mean is automatically identified as is the three 
sigma boundary.

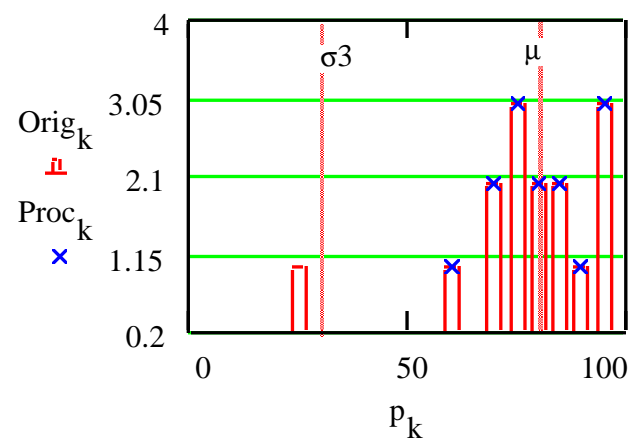

\section{Thermocouples}

Throughout the semester, the Mathcad documents were presented as tools for the design of an experiment as well as planning for analysis of the data after it was acquired. Since the Mathcad documents were live, emphasis could be placed on immediate analysis of data and preliminary interpretation of results

for real time adjustment of testing procedures. This feature was most evident in thermocouple measurements using the National Instruments SCXI-1121 data acquisition system which provided data to the same computer on which the Mathcad analysis document was resident. Using a large screen monitor, it was possible to take data, write it to a file, switch applications to Mathcad, and immediately calculate and display results including comparison of the theory with data. The example shown below was taken from a laboratory in which the students were required to determine the time constant associated with the response of a first-order measurement system, a thermocouple, to a step input, i.e. moving the thermocouple quickly from one temperature water to another.

The response of a first-order system to a step input was presented:

$$
y(t)=\kappa \cdot A+\left(y_{o}-\kappa \cdot A\right) \cdot e^{-\frac{t}{\tau}}
$$

The error function, gamma, was defined and the natural log of the error function was introduced, since, ultimately a linear relationship between time and log temperature was to be examined.

$$
\Gamma=e^{-\frac{t}{\tau}}=\frac{y(t)-\kappa \cdot A}{y_{0}-\kappa \cdot A} \quad \ln \left(\frac{-y(t)+\kappa \cdot A}{-y_{0}+\kappa \cdot A}\right)=-\frac{t}{\tau}
$$

Dummy indices were established to define a time variable and the total number of data points to be read from a Labview generated data file.

$$
\begin{aligned}
& \mathrm{N}:=400 \quad \mathrm{i}:=1 . . \mathrm{N} \\
& \mathrm{y}=\operatorname{READPRN}(\mathrm{CTH})
\end{aligned}
$$


The data was plotted to ensure that it was read properly and to identify the region in time over which the thermocouple responded. Then, a series of Mathcad statements were executed in order to determine the time constant tau, by determining the slope of the natural log of the error fraction.

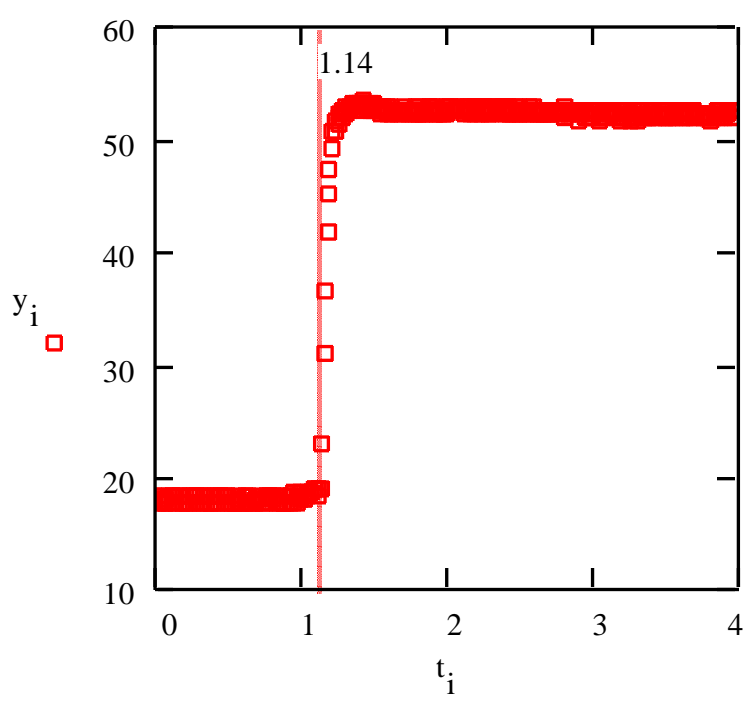

Here, an example of moving the thermocouple from cold to hot water is displayed. Additional dummy indices were required in order to sample the temperature data and establish the initial and steady state temperatures.

$$
\begin{aligned}
& \mathrm{j}:=21 . .40 \quad \mathrm{~s}:=\mathrm{N}-100 . . \mathrm{N} \\
& \mathrm{y}_{\text {initial }_{\mathrm{j}}:=\mathrm{y}_{\mathrm{j}}} \quad \mathrm{y}_{\text {steady }_{\mathrm{s}}}:=\mathrm{y}_{\mathrm{s}}
\end{aligned}
$$

These data were then averaged.

$$
\begin{aligned}
& y_{0}:=\frac{\sum_{j} y_{\text {initial }_{j}}}{20} \quad y_{s t}:=\frac{\sum_{\mathrm{s}} \mathrm{y}_{\text {steady }_{\mathrm{S}}}}{100} \\
& \mathrm{y}_{\mathrm{o}}=17.988 \quad \mathrm{y}_{\text {st }}=52.725
\end{aligned}
$$

Based on an examination of the data plot, other dummy indices were defined in order to segregate that part of the file which was an exponential response.

$$
\begin{aligned}
& \mathrm{n}:=114 \mathrm{Nn}:=122 \quad \mathrm{k}:=\mathrm{n} . . \mathrm{Nn} \\
& \text { ErrFraction }_{\mathrm{k}}:=\left(\frac{-\mathrm{y}_{\mathrm{k}}+\mathrm{y}_{\mathrm{st}}}{-\mathrm{y}_{\mathrm{o}}+\mathrm{y}_{\mathrm{st}}}\right)
\end{aligned}
$$




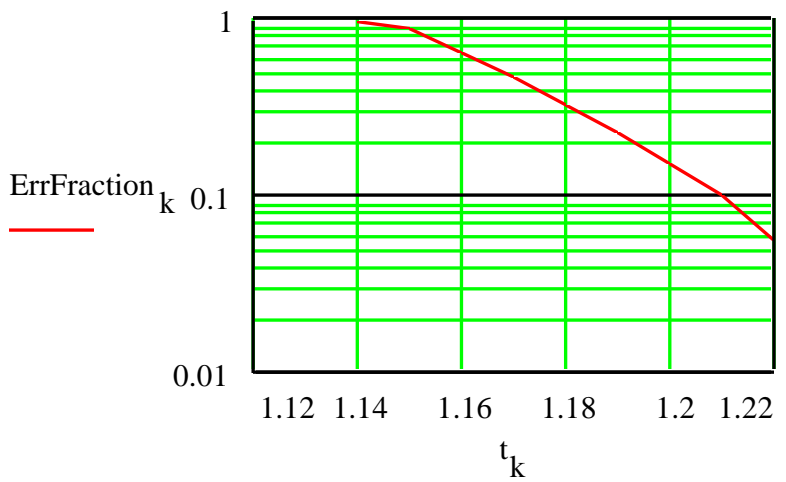

This graph was used to ensure that the data region which was selected approached a linear plot. Then, the slope of the line was calculated using a linear least squares fit. The range of the dependent and independent variables and the number of points to be used in the fit were determined.

$$
\begin{aligned}
& \operatorname{lny}_{\mathrm{k}}:=\ln \left(\text { ErrFraction }_{\mathrm{k}}\right) \quad \mathrm{tt}_{\mathrm{k}}:=\mathrm{t}_{\mathrm{k}} \\
& \mathrm{NN}:=(\mathrm{Nn}-\mathrm{n})+1
\end{aligned}
$$

The slope was calculated and used to calculate tau.

$$
\begin{gathered}
\mathrm{a} 1:=\frac{\sum \mathrm{tt} \cdot \sum \operatorname{lng}-\mathrm{NN} \cdot \sum_{\mathrm{k}} \mathrm{tt}_{\mathrm{k}} \cdot \operatorname{lng}_{\mathrm{k}}}{\left(\sum \mathrm{tt}\right)^{2}-\mathrm{NN} \cdot \sum_{\mathrm{k}}\left(\mathrm{tt}_{\mathrm{k}}\right)^{2}} \\
\tau:=-\frac{1}{\mathrm{a} 1} \quad \tau=0.028
\end{gathered}
$$

As a final check, the calculated tau was used in the system response and plotted against the data. In the plot below one more dummy index is used to displace the data file to coincide with the same zero time mark as the system response.

$$
\begin{aligned}
\text { SysRes }_{\mathrm{i}} & :=\mathrm{y}_{\mathrm{st}}+\left(\mathrm{y}_{\mathrm{o}}-\mathrm{y}_{\mathrm{st}}\right) \cdot \mathrm{e}^{-\frac{\mathrm{t}_{\mathrm{i}}}{\tau}} \\
\delta_{\mathrm{t}} & :=114 \quad \mathrm{i}:=1 . . \mathrm{N}-\delta \mathrm{t}
\end{aligned}
$$




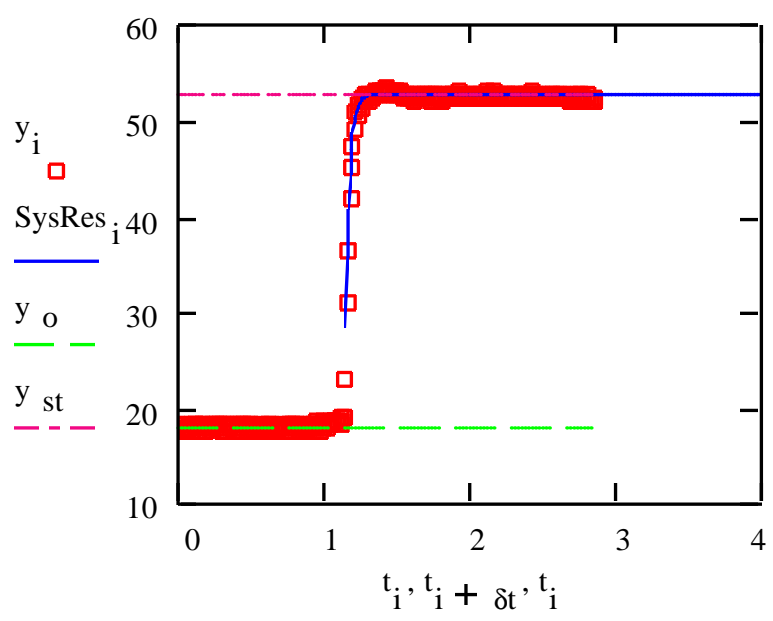

The responsiveness of this approach can not be given justice in written form, for the measurements and analysis occur virtually in real time, giving the student immediate feedback and the opportunity to inspect the data and repeat or change the measurement, if necessary.

\section{Periodic Time Series Data}

Perhaps one of the most powerful uses for Mathcad when tied to LabView is in the instruction of the analysis of periodic time series data. Once again, using a projection system or large screen monitor, the entire class can be exposed to the collection and analysis of data, the simulation of data and analysis, a demonstration of sampling theory, the impact of ensemble averaging, and the calculation of Fourier coefficients or Digital Fourier Transforms (DFT). The interactive nature of Mathcad and the flexibility of LabView as a data acquisition system provide a unique opportunity to allow students to write analysis algorithms. The work reported here was limited to instructor led discussions of an analysis approach. Students were encouraged to develop an analysis approach prior to acquiring data. While they did not have Mathcad available, they had the opportunity to execute their routines using Excel. In fact, the students were given an assignment to develop a routine to calculate a DFT of a sine wave during lecture. The students were banned from using intrinsic functions. The author's experience in this course as well as previous efforts (Ref 1) supports the assertion that, Mathcad, with its ability to handle complex math, symbolic manipulation, and, in the case of Mathcad 6.0, programming loops, offers a powerful and extremely flexible tool with which to explore and examine periodic data. The examples that follow were generated with Mathcad 3.0 but still provide evidence in support of this assertion.

\section{Sampling Theory}

There are two simple ways to demonstrate the effects of sampling using LabView and Mathcad. The first way was the method used in the EMFTS course, the second approach is an obvious extension which takes advantage of manipulating indices, which can be used in future courses, and provides useful insight.

A LabView virtual instrument (VI) locally designed was convenient for demonstrating sampling by permitting the students to vary sampling rate in real time. Simultaneous time and frequency plots changed as the student selected different sampling rates for capturing an externally generated sine wave. The Mathcad document used to enhance this student activity, did not directly use the LabView generated data, although this is a straightforward and simple extension.

A 1024 point 10 Hertz sine wave was generated in software and a sample interval established. 


$$
\begin{aligned}
& \mathrm{N}:=1024 \quad \mathrm{~F}_{\text {sample }}:=200 \\
& \mathrm{i}:=1 . . \mathrm{N}-1 \quad \delta \mathrm{t}:=\frac{1}{\mathrm{~F}_{\text {sample }}} \quad \mathrm{f}:=10 \text { Hertz } \quad \mathrm{t}_{\mathrm{i}}:=\mathrm{i} \cdot \delta \mathrm{t} \\
& \mathrm{k}:=1 . . \frac{\mathrm{N}}{2} \quad \text { Freq }:=\mathrm{fft}(\text { Sine }) \quad \mathrm{f}_{\mathrm{i}}:=\frac{\mathrm{k}}{\mathrm{N}} \cdot \mathrm{F} \text { sample }
\end{aligned}
$$
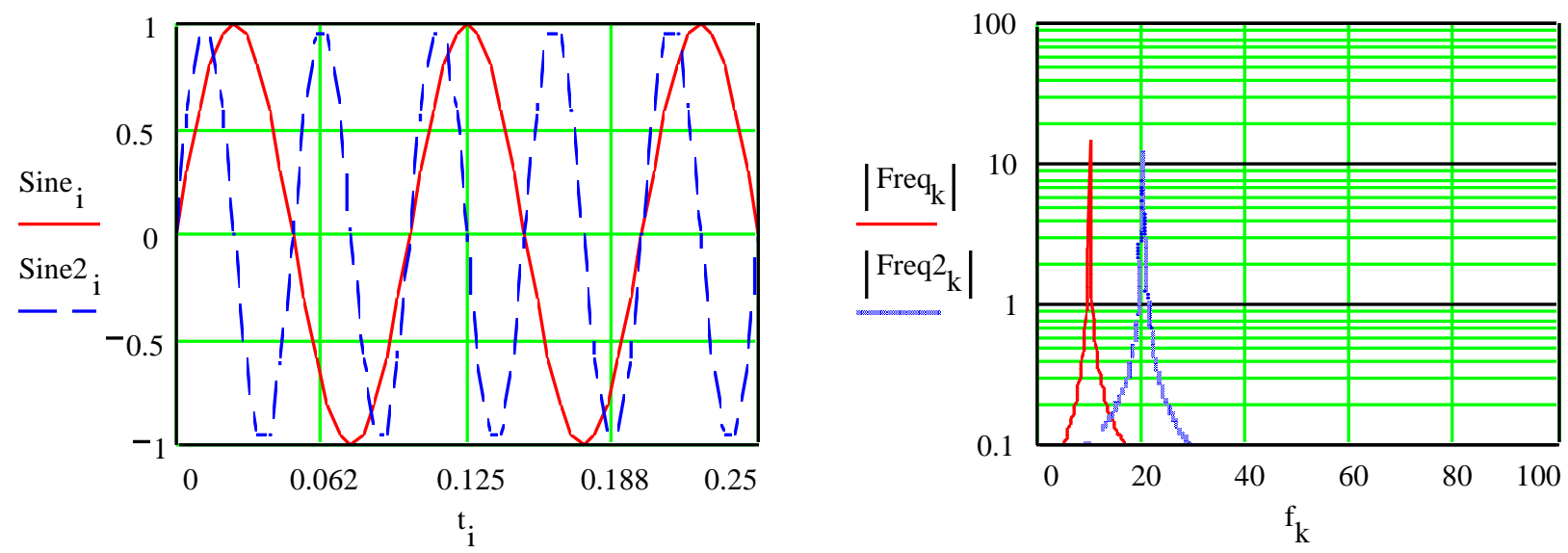

The time and frequency nature of the signals were displayed for the students. The instructor can vary the frequency, $\mathrm{f}$, between zero and half the sampling frequency to demonstrate the frequency content of the time series. To save space, two such cases have been shown here. However, it is a more effective and powerful presentation to change frequencies and watch the graphs update in real time using the large screen monitor.

Once the students are exposed to the time and frequency plots, sampling is easily demonstrated by generating a sine wave at a high frequency, but using the same time base, or sampling rate to display that sine wave.

$$
\begin{aligned}
\mathrm{f}_{\mathrm{h}} & :=175 \text { Hertz } \\
\mathrm{tt}_{\mathrm{i}}:=\frac{\mathrm{i} \cdot \delta \mathrm{t}}{10} & \text { Sampled }_{\mathrm{i}}:=\sin \left(2 \cdot \pi \cdot \mathrm{f}_{\mathrm{h}} \cdot \mathrm{tt}_{\mathrm{i}}\right) \\
\mathrm{t}_{\mathrm{i}}:=\mathrm{i} \cdot \delta \mathrm{t} & \text { UnderSamr }_{\mathrm{i}}:=\sin \left(2 \cdot \pi \cdot \mathrm{f}_{\mathrm{h}} \cdot \mathrm{t}_{\mathrm{i}}\right)
\end{aligned}
$$

The student can readily see that the analytical expressions for the two waves are the same, but that the time base, or the rate at which samples of these waves are taken, are different by a factor of ten. A powerful comparison can be achieved by simply plotting a properly sampled 175 Hertz wave on the same graph as the under sampled version as shown. Once again, the interactive nature of Mathcad can be exploited. 


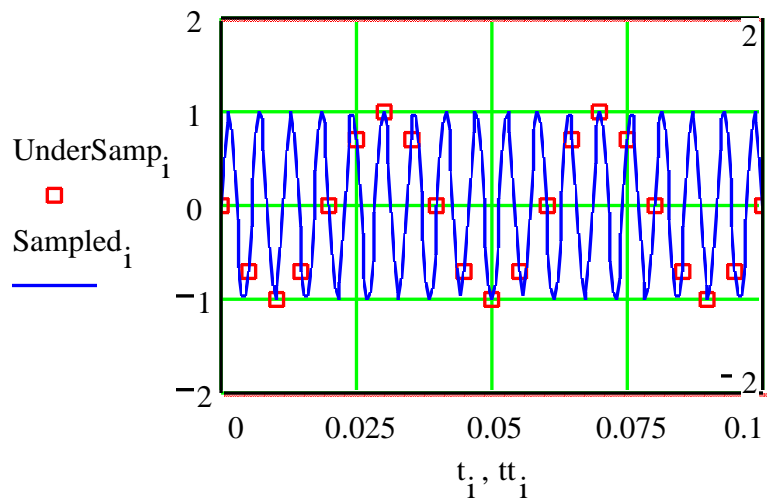

The frequency of the under sampled sine wave, demonstrates that the $175 \mathrm{~Hz}$ wave is clearly aliased and appears at the "folded" frequency of $25 \mathrm{~Hz}$.

The second approach to examining the effects of sampling, which was alluded to earlier, was not used in this course, but was uncovered in the course of the development of the material for the EMFTS course. Here, a data file was generated by an appropriate LabView VI, a simple sine wave generator. Once the file was written, it was easily read by Mathcad for display and manipulation purposes as shown.

This approach is simple, yet powerful. By changing the parameter, s, the student effectively could change the step at which data is plotted, and thus, sampled. The data file to which LabView wrote data still has all the data points available, the student simply selects the discrete steps at which the data is written. Once again, the interactive nature of Mathcad and a projection system would allow the instructor to vary the step size and instantly demonstrate the effects of aliasing.

$$
\begin{aligned}
& \mathrm{y}:=\operatorname{READPRN}(\text { sine }) \\
& \mathrm{s}:=64 \quad \mathrm{j}:=0, \mathrm{~s} . . \mathrm{N}-1
\end{aligned}
$$

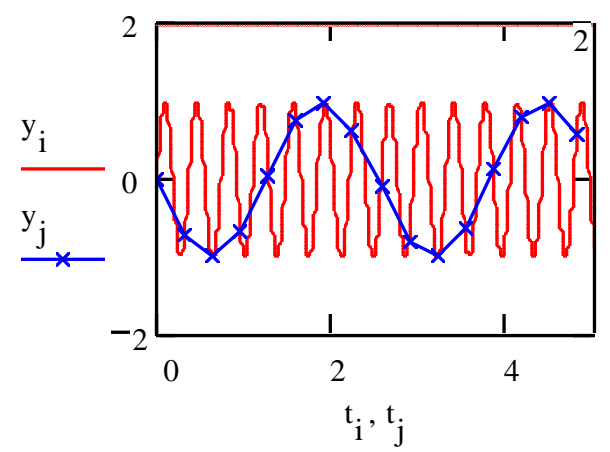

The graph above shows the original data file, that is, every data point taken by LabView, and the under sampled wave driven by the selection of the step size, $s$.

\section{Ensemble Averaging}

During the Fourier analysis portion of the EMFTS course, the effect of noise on the time and frequency representations of a signal was examined using a LabView VI. While it would have been possible to write a number of time series to disk using LabView for the purpose of ensemble time averaging, Mathcad was employed for this portion of the demonstration since it was more expedient. Here, five separate 15 Hertz sine waves with uniform random noise were generated using Mathcad. 
Uniform noise was used here, but Mathcad 6.0 allows for a variety of random number distributions including Gausian, which would be more representative of a physical system. The author has verified the simplicity with which different noise distributions can be used in 6.0. The Mathcad document presented here, was used during the course. The parameters below define the radian frequency, time resolution, the noise amplitude, the number of data points, dummy indices, frequency, and total elapsed time.

$$
\begin{aligned}
& y 1_{i}:=\sin \left(\omega \cdot t_{i}\right)+\operatorname{rnd}(n)-\frac{n}{2} \\
& y 2_{i}:=\sin \left(\omega \cdot t_{i}\right)+\operatorname{rnd}(n)-\frac{n}{2} \\
& \mathrm{y} 3_{\mathrm{i}}:=\sin \left(\omega \cdot \mathrm{t}_{\mathrm{i}}\right)+\operatorname{rnd}(\mathrm{n})-\frac{\mathrm{n}}{2} \quad \omega:=2 \cdot \pi \cdot 15 \quad \delta \mathrm{t}:=.01 \quad \mathrm{n}:=5 \\
& \mathrm{y}_{\mathrm{i}}:=\sin \left(\omega \cdot \mathrm{t}_{\mathrm{i}}\right)+\operatorname{rnd}(\mathrm{n})-\frac{\mathrm{n}}{2} \quad \mathrm{~N}:=256 \quad \mathrm{i}:=1 . . \mathrm{N} \quad \mathrm{k}:=1 . .128 \\
& \mathrm{y} 5_{\mathrm{i}}:=\sin \left(\omega \cdot \mathrm{t}_{\mathrm{i}}\right)+\operatorname{rnd}(\mathrm{n})-\frac{\mathrm{n}}{2} \quad \mathrm{f}_{\mathrm{k}}:=\frac{\mathrm{k}}{\mathrm{N} \cdot \delta \mathrm{t}} \quad \mathrm{t}_{\mathrm{i}}:=\mathrm{i} \cdot \delta \mathrm{t}
\end{aligned}
$$

The five wave forms above can be viewed as independent time series of a 15 Hertz wave; examples using three noise amplitudes are shown.

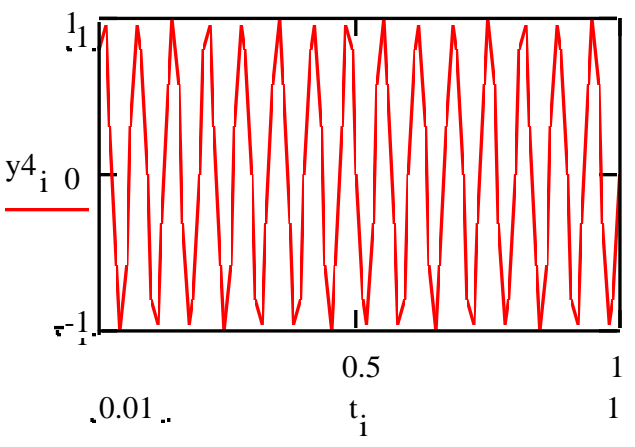

No Noise $\mathbf{n}=\mathbf{0}$

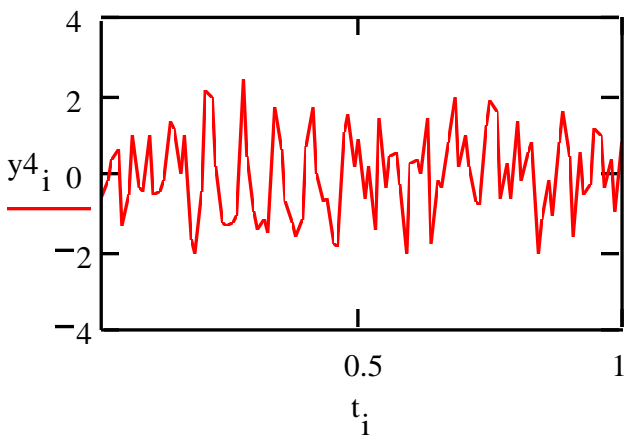

Moderate Noise $n=3$

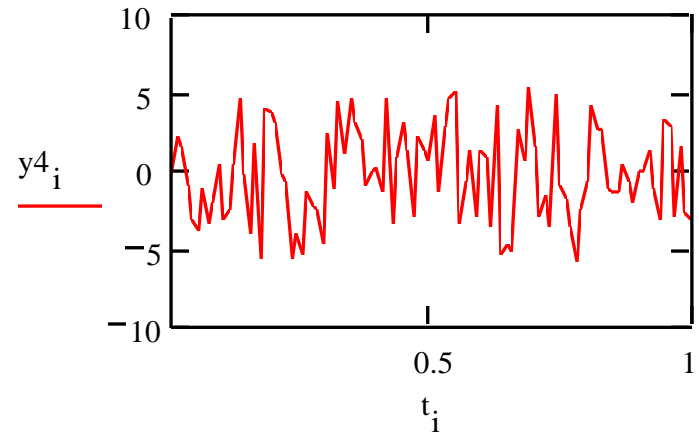

High Noise $n=10$

These three graphs show the apparent disappearance of any periodicity in the data. Students were then shown, through the Digital Fourier Transform (DFT), that the signal level was still present. 

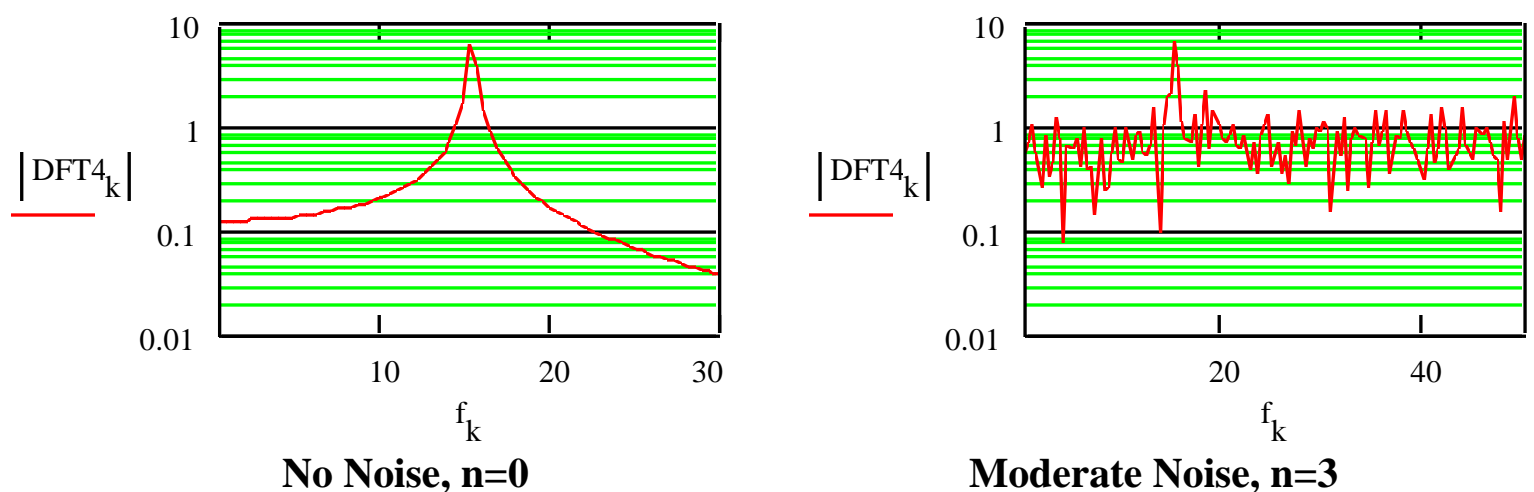

Moderate Noise, $n=3$

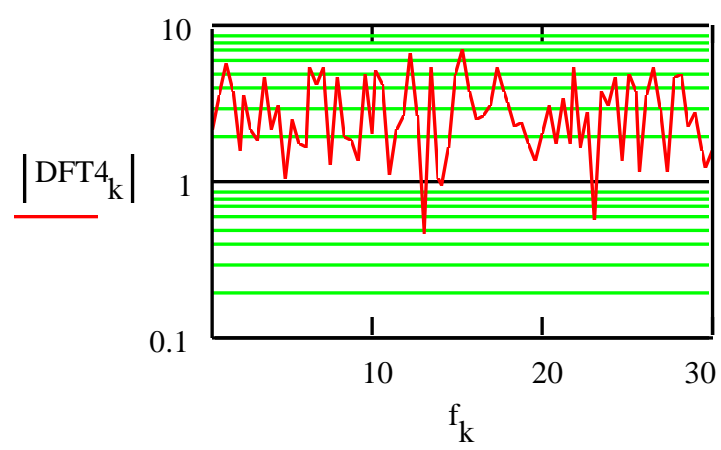

High Noise, $\mathrm{n}=10$

Through an examination of these DFT plots the students can gain an appreciation of the fact that the signal is still present, but that the noise level has increased. The random nature of the noise then, provides an excellent opportunity to discuss ensemble averaging. In the EMFTS course here, time constraints prohibited an in-depth discussion and the presentation was limited to a demonstration to approximately half of the class.

Using the definition of time series ensemble averaging (Ref 2),the value of averaging was demonstrated.

$$
\mathrm{y}_{\mathrm{ave}_{\mathrm{i}}}:=\left(\frac{\mathrm{y} 1+\mathrm{y} 2+\mathrm{y} 3+\mathrm{y} 4+\mathrm{y} 5}{5}\right)_{\mathrm{i}}
$$

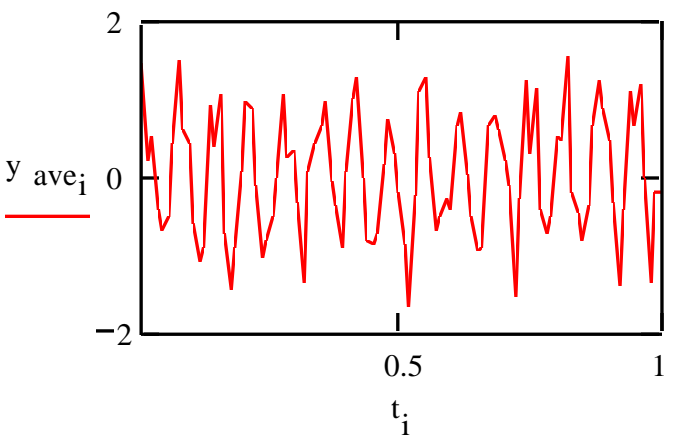

Moderate Noise, $\mathbf{n}=3$

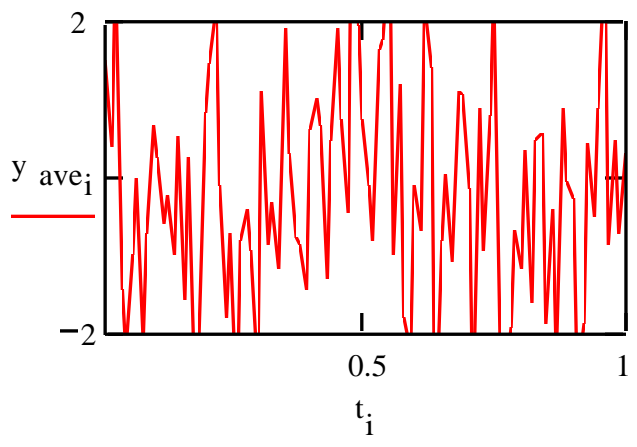

High Noise, $\mathbf{n = 1 0}$ 
In the moderate noise case, the students could see that the periodicity was still apparent in the time plot. Using the DFT, they could see that the spectral noise level dropped in these cases as compared to their non-averaged counterparts, and that the periodic signal was still present and obvious, even in the high noise case.
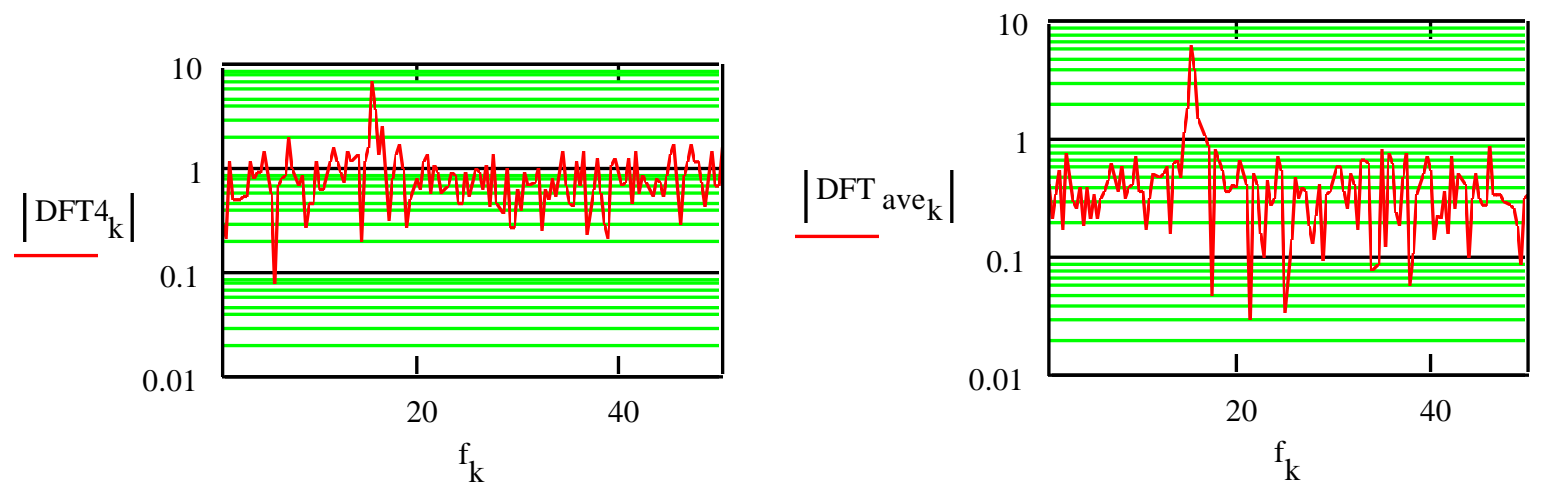

Moderate Noise, $\mathbf{n}=3$
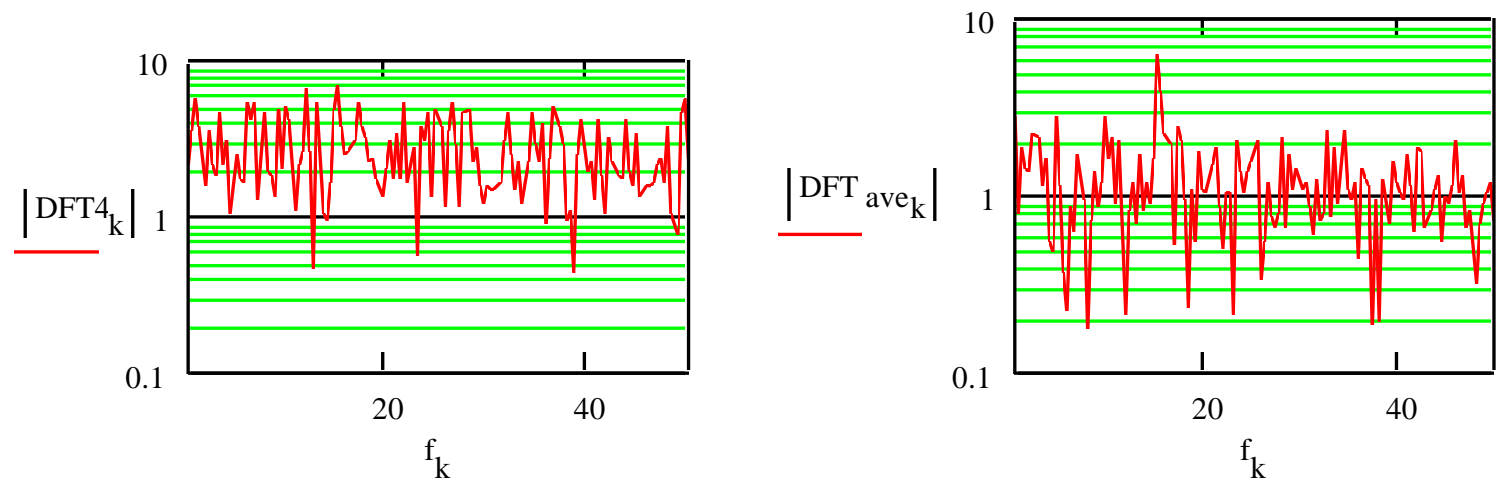

High Noise, $\mathbf{n}=10$

In each example shown, the individual DFT and the DFT of the time average are shown on separate plots for clarity. Once again, the use of a projection system or large screen monitor greatly enhances and expedites the presentation.

\section{Fourier Coefficients and the DFT}

An important part of the EMFTS course was the lectures and laboratories describing the analysis of time periodic signals using Fourier analysis. Students were introduced to Fourier Series in the traditional way and were asked to calculate the Fourier coefficients for a periodic function, in a laboratory. This particular laboratory was one of the more creative and popular modules. In this laboratory, students were tasked with making a lighthouse of particular period using five available "signal generators". The signal generators were actually wave form generators resident in LabView. The VI developed for this lab allowed students to input the individual wave form amplitudes and frequencies for each of the five wave form generators, which were added, in turn, to present the final output. The output was in the form of an analog signal from the National Instruments system which powered an LED. Thus, the student not only experienced calculating the proper coefficients but actually lit the "lighthouse".

The contribution made in software for this laboratory was in keeping with the intent of the previous modules. The equations for the periodic signal were determined and displayed, and the Fourier 
coefficients were determined symbolically and numerically. It was impossible to use this Mathcad document as a demonstration for the students without revealing the solution, so that the document was used as an additional aid in reviewing the solution and in examining the impact of changing the number of sine terms retained in the solution. Student solutions were done with hand-held calculators; the intent here was to model and reinforce the value of prior planning and analysis.

The desired signal was a square wave with a four second period which oscillated about a 1.5 volt DC offset. It is shown below as generated in Mathcad. Mathcad offers the Heaviside function as an intrinsic function, as well.

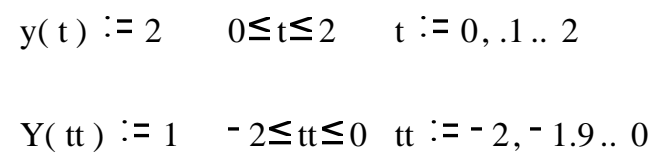

$$
\begin{aligned}
& \mathrm{A}_{0}=\frac{1}{\mathrm{~T}} \cdot \int_{-\frac{\mathrm{T}}{2}}^{\frac{\mathrm{T}}{2}} \mathrm{f}(\mathrm{t}) \mathrm{dt} \quad \mathrm{T}:=4 \\
& \mathrm{~A}_{0}:=\frac{1}{\mathrm{~T}} \cdot\left[\int_{-\frac{\mathrm{T}}{2}}^{0} 1 \mathrm{dt}+\int_{0}^{\frac{\mathrm{T}}{2}} 2 \mathrm{dt}\right] \\
& \mathrm{A}_{0}=1.5
\end{aligned}
$$

Almost invariably, the students' initial reaction was to treat the step function as an odd function, which led them away from calculating the Ao term.

The remaining A terms do indeed go to zero as is shown easily in Mathcad through symbolic integration.

$A_{n}=\frac{2}{T} \cdot\left[\int_{-\frac{T}{2}}^{0} \cos (n \cdot \omega \cdot t) d t+\int_{0}^{\frac{T}{2}} 2 \cdot \cos (n \cdot \omega \cdot t) d t\right]$

The period was given as four seconds and the definition of the radian frequency was used to reduce the expression to one which the students could see must be zero for all $\mathrm{n}$.

$$
\begin{aligned}
& A_{n}=\frac{6}{T} \cdot \frac{\sin \left(\frac{1}{2} \cdot n \cdot \omega \cdot T\right)}{n \cdot \omega} \quad \text { where } \omega=\frac{2 \cdot \pi}{T} \\
& A_{n}=3 \cdot \frac{\sin (n \cdot \pi)}{n \cdot \pi}
\end{aligned}
$$

The B terms were evaluated in the same fashion. 


$$
B_{n}=\frac{2}{T} \cdot\left[\int_{-\frac{T}{2}}^{0} \sin (n \cdot \omega \cdot t) d t+\int_{0}^{\frac{T}{2}} 2 \cdot \sin (n \cdot \omega \cdot t) d t\right]
$$

$$
\begin{aligned}
& B_{n}=\frac{2}{T} \cdot\left(\frac{1}{n \cdot \omega}-\frac{\cos \left(\frac{1}{2} \cdot n \cdot \omega \cdot T\right)}{n \cdot \omega}\right) \\
& B_{n}=\frac{2}{T} \cdot\left(\frac{1}{2 \cdot n \cdot \pi} \cdot T-\frac{1}{2} \cdot \frac{\cos (n \cdot \pi)}{n \cdot \pi} \cdot T\right)
\end{aligned}
$$

The resulting expression for the coefficients was used as a live expression in Mathcad in an evaluation of the Fourier series of the original step function.

$$
\begin{aligned}
y(t):= & \sum_{n} \frac{2}{T} \cdot\left(\frac{1}{2 \cdot n \cdot \pi} \cdot T-\frac{1}{2} \cdot \frac{\cos (n \cdot \pi)}{n \cdot \pi} \cdot T\right) \cdot \sin \left(n \cdot \pi \cdot 2 \cdot \frac{t}{T}\right) \ldots \\
& +A_{0}
\end{aligned}
$$

This expression was graphed as a function of time with a variety of values for $\mathrm{n}$, the number of terms. Thus, it is trivial to show students, or better yet, have students experience through interaction with the equation, the effect of the number of terms on the fidelity of the output. For demonstration purposes, twenty-six terms are displayed here.

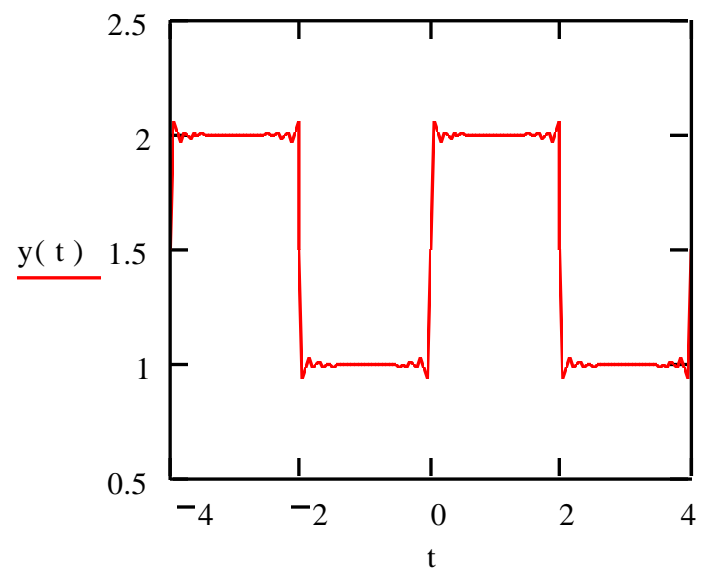

The actual solution to the lighthouse was then readily available to the student. Mathcad allows the student, and in our case the instructor, to display the values of the coefficients, the frequencies and the waveform. 


$$
B_{n}:=\frac{2}{T} \cdot\left(\frac{1}{2 \cdot n \cdot \pi} \cdot T-\frac{1}{2} \cdot \frac{\cos (n \cdot \pi)}{n \cdot \pi} \cdot T\right)
$$

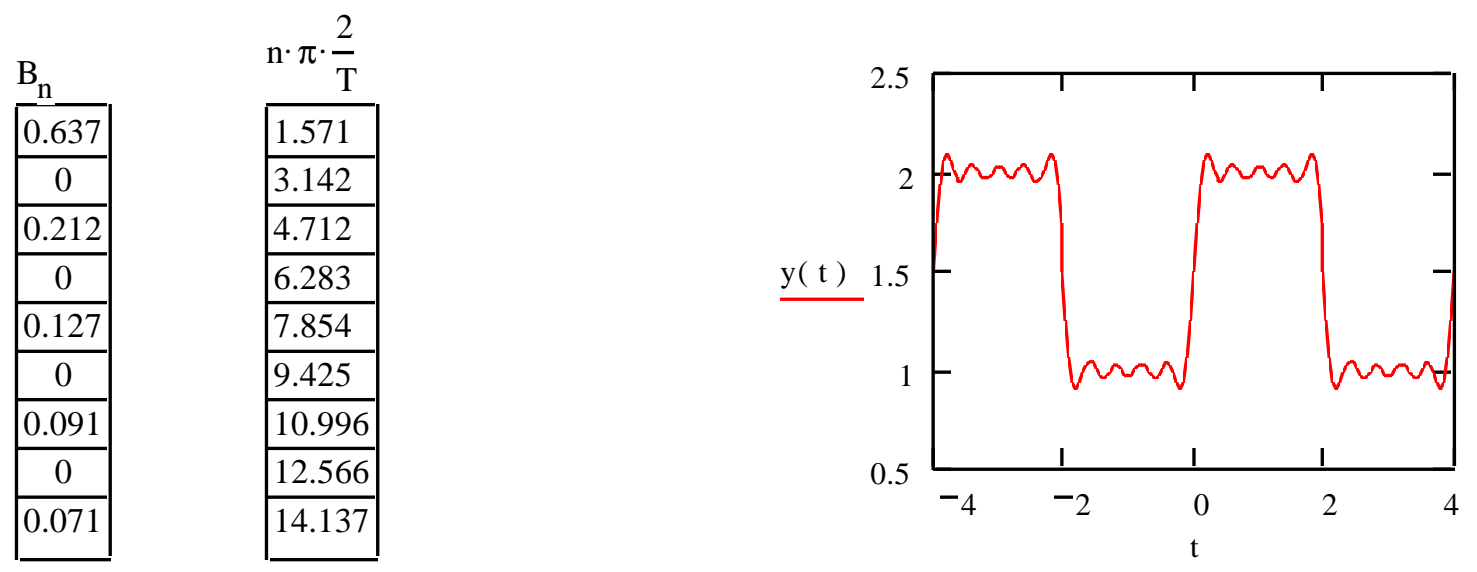

In the lab, the students actually could see the effect of the Gibbs effect as the LEDs brightened and dimmed in response to the varying voltages.

\section{CONCLUSIONS}

Mathcad as a stand-alone equation solving and spreadsheet software is an exceptional tool for use in engineering education. LabView, while primarily a research or operational grade data acquisition system, has been adapted well for educational use. The combination of these two computer-based tools provides an elegant and powerful option to the instructor for acquiring and analyzing data in real time. The efficiency in presentation, the complexity of problems that can be addressed, the ease with which data can be acquired and analyzed, and the degree to which the structured thought required to design the Mathcad documents mirrors that of algorithm development all contribute to a richer educational experience for the students.

One negative aspect of using these tools lies in the potential to superficially treat fundamental mathematics in an attempt to address sophisticated data acquisition laboratories. The instructor must take care to highlight the fundamental mathematics and use Mathcad to facilitate that presentation. This can be done, by assignments to the student that accentuate the fundamentals while still allowing the students to avail themselves of the advantages of these tools. A case in point, involves the generation of DFTs. Students in this course were given the assignment to write a program in any computer language to calculate a 32 point DFT. The students did not have Mathcad or LabView available to them. However, in the future, this assignment could be executed in a variety of ways with Mathcad, capitalizing on its ability to easily handle complex math: straightforward evaluation of the analytical expression for a DFT as presented in the text $(\operatorname{Ref} 3)$,

$$
c_{k}:=\frac{2}{n} \cdot \sum_{r} y_{r} \cdot e^{2 \cdot \pi \cdot i \cdot \frac{k}{n} \cdot r}
$$

expansion of this expression using Euler's formula, or, in the case of Mathcad 6.0, evaluation of the DFT using a "for" loop. The students could, obviously, check their results with the intrinsic functions available to them in Mathcad.

\section{ACKNOWLEDGEMENT}


The author would not have been able to generate the Mathcad analysis modules included in this paper without the freedom and support afforded him by LCDR. V. Wilczynski and Prof. Gregg Dixon of the

Mechanical Engineering Section of the USCGA. In addition, much of the work here was modeled after existing LabView VIs generated by LCDR. Wilczynski.

\section{REFERENCES}

1) Walters, David L., "The Use of Mathcad and Autocad to Enhance Instruction of Dynamics, Theory of Machines and Vibrations", Proceedings of the ASEE Gulf-Southwest Section Meeting, Beaumont, TX March 1995

2) Bendat, Julius S. and Piersol, Allan G. Engineering Applications of Correlation and Spectral Analysis John Wiley \& Sons 1980

3) Figliola, Richard S. and Beasley, Donald E., Theory and Design for Mechanical Measurements, 2nd Ed. John Wiley \& Sons 1995 